\title{
On skew-symmetric differentiation matrices
}

\author{
Arieh Iserles
}

January 9, 2013

\begin{abstract}
The theme of this paper is the construction of finite-difference approximations to the first derivative in the presence of Dirichlet boundary conditions. Stable implementation of splitting-based discretisation methods for the convectiondiffusion equation requires the underlying matrix to be skew symmetric and this turns out to be a surprisingly restrictive condition. We prove that no skewsymmetric approximation on an equidistant grid may exceed order two. Once non-equidistant grid is allowed, this barrier can be breached.
\end{abstract}

\section{Introduction}

The theory of finite-difference methods for discretised partial differential equations is mature and well-understood, the mainstay of numerous computational algorithms for the past century. It is a sobering fact however that, even in its basic linear setting, this theory is incomplete. The theme of this paper is an interesting lacuna in the classical theory.

A good starting point is the convection-diffusion equation (Bejan 2004, Morton 1996) in a univariate setting,

$$
\frac{\partial u}{\partial t}=\frac{\partial^{2} u}{\partial x^{2}}+v(x) \frac{\partial u}{\partial x}, \quad t \geq 0, \quad x \in[-1,1]
$$

accompanied by an initial condition for $t=0$ and Dirichlet boundary conditions at $x=0$ and $x=1$, where the average transport velocity $v$ is a given smooth function. The equation (1.1) is important because its multivariate generalisation,

$$
\frac{\partial u}{\partial t}=\nabla^{2} u+\boldsymbol{v}(\boldsymbol{x}) \cdot \boldsymbol{\nabla} u, \quad t \geq 0, \quad \boldsymbol{x} \in[0,1]^{d},
$$

is a model for transport phenomena combining diffusive and convective processes.

A powerful means to solve (1.1), easily extended to a multivariate setting, is through an exponential splitting method (McLachlan \& Quispel 2002). Its simplest manifestation, the Strang splitting, approximates (1.1) by the time-stepping scheme

$$
\boldsymbol{u}^{n+1}=\mathrm{e}^{\frac{1}{2}(\Delta t) C} \mathrm{e}^{(\Delta t) \mathcal{D}_{2}} \mathrm{e}^{\frac{1}{2}(\Delta t) C} \boldsymbol{u}^{n}+\boldsymbol{f}^{n}, \quad n \geq 0,
$$

where $u_{m}^{n} \approx u(m \Delta x, n \Delta t), \mathcal{D}_{2}$ is a second differentiation matrix (that is, an approximation to the second derivative using a linear combination of grid points), while $C$ 
is the outcome of multiplying each $m$ th row of the first differentiation matrix $\mathcal{D}_{1}$, say, by $v(m \Delta x)$. The inhomogeneous vector $\boldsymbol{f}^{n}$ consists of boundary contributions (Hochbruck \& Ostermann 2010, Iserles 1992) and we note that $\boldsymbol{f}^{n} \equiv \mathbf{0}$ when the Dirichlet boundary conditions are zero. Since

$$
\mathrm{e}^{\frac{1}{2}(\Delta t) C} \mathrm{e}^{(\Delta t) \mathcal{D}_{2}} \mathrm{e}^{\frac{1}{2}(\Delta t) C}=\mathrm{e}^{(\Delta t)\left(\mathcal{D}_{2}+C\right)}+\mathcal{O}\left((\Delta t)^{3}\right),
$$

the method is second order in time, therefore it makes sense to discretise in space to a similar order - this is the case with the familiar differentiation matrices

$$
\mathcal{D}_{1}=\frac{1}{\Delta x}\left[\begin{array}{ccccc}
0 & \frac{1}{2} & 0 & \cdots & 0 \\
-\frac{1}{2} & 0 & \frac{1}{2} & \ddots & \vdots \\
0 & \ddots & \ddots & \ddots & 0 \\
\vdots & \ddots & \ddots & \ddots & \frac{1}{2} \\
0 & \cdots & 0 & -\frac{1}{2} & 0
\end{array}\right], \quad \mathcal{D}_{2}=\frac{1}{(\Delta x)^{2}}\left[\begin{array}{ccccc}
-2 & 1 & 0 & \cdots & 0 \\
1 & -2 & 1 & \ddots & \vdots \\
0 & \ddots & \ddots & \ddots & 0 \\
\vdots & \ddots & \ddots & \ddots & 1 \\
0 & \cdots & 0 & 1 & -2
\end{array}\right] .
$$

We do not consider in this paper the practicalities of computing the exponentials in (1.2), whether exactly or by some sort of approximation.

Inasmuch as higher-order splitting methods are available, mostly in the context of symplectic integration and the solution of hyperbolic PDEs (Hairer, Lubich \& Wanner 2006, McLachlan \& Quispel 2002), the Strang splitting is widely accepted as the best of its kind insofar as parabolic PDEs are concerned, because a theorem of Sheng (1989) indicates that no stable splitting for such equations may exceed order 2. However, the universality of the Sheng barrier has been recently challenged from three different quarters. Firstly, a new breed of ODE solvers, exponential integrators, have been methodically applied to semidiscretised PDEs, allowing for the use of high-order time discretisation, combined with the use of exponential functions (Hochbruck \& Ostermann 2010). Secondly, (Hansen \& Ostermann 2009) have demonstrated that using complex-valued time it is possible to breach the Sheng barrier. Thirdly, work in progress demonstrates that the recent methodology of Zassenhaus splittings (Bader, Iserles, Kropielnicka \& Singh 2012) can be transplanted into the setting of the convection-diffusion equation (1.1) in a stable manner, while allowing for the use of higher-order splittings incorporating commutators and thereby being outside the terms of the Sheng Theorem.

All this motivates our interest in higher-order differentiation matrices. Specifically, we wish to approximate the first derivative to a higher order by a matrix $\mathcal{D}_{1}$. Yet, the constraint is that the matrix $C$, which we form from $\mathcal{D}_{1}$ multiplying each $m$ th row by $v(m \Delta x)$, results in a stable exponential. In other words, we wish for $\left\|\mathrm{e}^{n(\Delta t) C}\right\|$ to be uniformly bounded for $n \Delta t$ in a compact interval, while $\Delta x, \Delta t \rightarrow 0$ and the Courant number $\mu=\Delta t / \Delta x$ is constant (Iserles 2008, Richtmyer \& Morton 1967). In Section 2 we demonstrate that, subject to mild side conditions, a necessary and sufficient condition for stability is that the matrix $\mathcal{D}_{1}$ is skew symmetric. This condition is clearly satisfied by the matrix $\mathcal{D}_{1}$ that we have considered above but this, as turns out in Section 3, is an exception: there exist no third-order (or higher) skew-symmetric differentiation matrices!

Like any 'impossibility result', the order restriction on skew-symmetric differentiation matrices does not mean that it is impossible to use finite difference methods in 
this setting. All it means is that we must change some of our underlying assumptions. In Section 4 we show that, once the assumption that the grid points are equidistant is lifted, it is possible to breach the barrier and obtain a skew-symmetric matrix $\mathcal{D}_{1}$ corresponding to a third-order method.

It is important to emphasise that the absence of high-order skew-symmetric differentiation matrices is purely an artefact of Dirichlet boundary conditions. Once we consider periodic boundary conditions and are allowed to 'wrap around' the matrix $\mathcal{D}_{1}$, we can easily generate skew-symmetric matrices of any order! For example,

$$
u_{m}^{\prime} \approx \frac{1}{\Delta x}\left(\frac{1}{12} u_{m-2}-\frac{2}{3} u_{m-1}+\frac{2}{3} u_{m+1}-\frac{1}{12} u_{m+2}\right), \quad m=1, \ldots, M,
$$

where $\Delta x=1 / M$, represents a fourth-order scheme. Once we wrap around boundaries, i.e. identify $m$ with $m \bmod M$, the outcome is the skew-symmetric banded circulant

$$
\mathcal{D}_{1}=\frac{1}{\Delta x}\left[\begin{array}{cccccccc}
0 & \frac{2}{3} & -\frac{1}{12} & 0 & 0 & \ldots & \frac{1}{12} & -\frac{2}{3} \\
-\frac{2}{3} & 0 & \frac{2}{3} & -\frac{1}{12} & 0 & \ldots & 0 & \frac{1}{12} \\
\frac{1}{12} & -\frac{2}{3} & 0 & \frac{2}{3} & -\frac{1}{12} & 0 & \ldots & 0 \\
0 & \ddots & \ddots & \ddots & \ddots & \ddots & \ddots & \vdots \\
\vdots & \ddots & \ddots & \ddots & \ddots & \ddots & \ddots & 0 \\
0 & \ldots & 0 & \frac{1}{12} & -\frac{2}{3} & 0 & \frac{2}{3} & -\frac{1}{12} \\
-\frac{1}{12} & 0 & \ldots & 0 & \frac{1}{12} & -\frac{2}{3} & 0 & \frac{2}{3} \\
\frac{2}{3} & -\frac{1}{12} & 0 & \ldots & 0 & \frac{1}{12} & -\frac{2}{3} & 0
\end{array}\right] .
$$

An obvious (and often superior) alternative to finite differences are spectral methods. Here, again, periodic boundary conditions lead to skew-Hermitian matrices but the problem, once we consider Dirichlet boundary conditions, is if at all even more acute. The standard approach in that case is to use Chebyshev expansions (Hesthaven, Gottlieb \& Gottlieb 2007) but in that case - indeed, in the case of any polynomial basis - the differentiation matrix is necessarily lower triangular: as far away from skew symmetry as one can get. Given that generalising the non-equidistant approach of Section 4 to high orders is far from simple, it seems that no methodology to construct high order skew-symmetric differentiation matrices $\mathcal{D}_{1}$ is presently available.

\section{On stability and skew symmetry}

Let $u$ be a smooth function for $x \in[0,1]$, set $u_{m}=u(m \Delta x), u_{m}^{\prime}=u^{\prime}(m \Delta x)$ for $m=0,1, \ldots, M+1$, where $\Delta x=1 /(M+1)$ and consider the approximation

$$
u_{m}^{\prime} \approx \frac{1}{\Delta x} \sum_{k=0}^{M+1} b_{m, k} u_{k}, \quad m=1,2, \ldots, M .
$$


The underlying differentiation matrix ${ }^{1} \mathcal{D}_{1}$ excludes boundary conditions, thereby is of the form

$$
\mathcal{D}_{1}=\frac{1}{\Delta x} B, \quad \text { where } \quad B=\left[\begin{array}{cccc}
b_{1,1} & b_{1,2} & \cdots & b_{1, M} \\
b_{2,1} & b_{2,2} & \cdots & b_{2, M} \\
\vdots & \vdots & & \vdots \\
b_{M, 1} & b_{M, 2} & \cdots & b_{M, M}
\end{array}\right] .
$$

The coefficients $b_{m, k}$ may themselves depend on $M$ but, in the interests of clarity, we suppress this dependence in our notation.

The semidiscretisation (2.1) is stable (in the sense of Lax) if, assuming zero Dirichlet boundary conditions $u_{0}, u_{M+1} \equiv 0$, it produces a solution vector $\boldsymbol{u}(t)$ which is uniformly bounded in the $\ell_{2}$ norm in every compact time interval. As is well known, stability is equivalent to the statement that

$$
\left\|\mathrm{e}^{(\Delta t) /(\Delta x) B}\right\| \leq 1+\alpha \Delta t
$$

for some $\alpha \geq 0$, uniformly for $\Delta t, \Delta x \rightarrow 0$, provided that $\Delta t / \Delta x$ is bounded away from zero and infinity (Iserles 2008).

It is important to recall that, unless $B$ is a normal matrix, $(2.2)$ is not a statement on its eigenvalues: specifically, it is not sufficient for all the eigenvalues of $B$ to reside in a shifted complex left half plane. We must instead consider its logarithmic norm (Söderlind 2006) $\mu[B]$, namely the largest eigenvalue of the symmetric matrix $\tilde{B}=$ $\frac{1}{2}\left(B+B^{\top}\right)$. It is well known that

$$
\left\|\mathrm{e}^{t B}\right\| \leq \mathrm{e}^{t \mu[B]}, \quad t \geq 0,
$$

and that $\mu[B]$ is the least constant consistent with this inequality. Therefore, (2.2) is equivalent to the statement that $\mu[(\Delta t /(\Delta x) B] \leq \alpha \Delta t$, therefore $\mu[B] \leq \alpha \Delta x$, for some $\alpha \geq 0$.

Clearly, if $B$ is skew symmetric then $\mu[B]=0$ and stability is assured. Suppose however that for every $M$ we have $\mu[B]=\kappa_{M}, \kappa_{M}>0$, and that $\lim \sup _{M \rightarrow \infty} \kappa_{M}>0$. Then (2.1) is unstable. As an example, consider amending the differentiation matrix from (1.3) by replacing its first and last row by

$$
\begin{aligned}
u_{1}^{\prime} & \approx \frac{1}{\Delta x}\left(-\frac{1}{4} u_{0}-\frac{5}{6} u_{1}+\frac{3}{2} u_{2}-\frac{1}{2} u_{3}+\frac{1}{12} u_{4}\right), \\
u_{M}^{\prime} & \approx \frac{1}{(\Delta x}\left(-\frac{1}{12} u_{M-3}+\frac{1}{2} u_{M-2}-\frac{3}{2} u_{M-1}+\frac{5}{6} u_{M}+\frac{1}{4} u_{M+1}\right)
\end{aligned}
$$

respectively - note that order 4 is retained. (Because we are considering Dirichlet boundary conditions, $\Delta x=1 /(M+1)$.) We have

$$
\tilde{B}=\left[\begin{array}{ccc}
\tilde{B}_{1} & O & O \\
O & O & O \\
O & O & \tilde{B}_{2}
\end{array}\right],
$$

\footnotetext{
${ }^{1}$ The phrase "differentiation matrix" originates in spectral methods, where it denotes an exact linear relationship between basis functions and their derivatives. By rights, we should have called $\mathcal{D}_{1}$ an "approximate differentiation matrix" but we sacrifice nitpicking on the altar of brevity.
} 
where

$$
\tilde{B}_{1}=\left[\begin{array}{cccc}
-\frac{5}{6} & \frac{5}{12} & -\frac{5}{24} & \frac{1}{24} \\
\frac{5}{12} & 0 & 0 & 0 \\
-\frac{5}{24} & 0 & 0 & 0 \\
\frac{1}{24} & 0 & 0 & 0
\end{array}\right], \quad \tilde{B}_{2}=\left[\begin{array}{cccc}
0 & 0 & 0 & -\frac{1}{24} \\
0 & 0 & 0 & \frac{5}{24} \\
0 & 0 & 0 & -\frac{5}{12} \\
-\frac{1}{24} & \frac{5}{24} & -\frac{5}{12} & \frac{5}{6} .
\end{array}\right] .
$$

Thus, $\tilde{B}$ departs from zero in just eight columns: actually, it is a rank-4 matrix whose nonzero eigenvalues are $\pm \frac{5}{12} \pm \frac{\sqrt{226}}{24}, \kappa_{M} \equiv \mu[B]=\frac{5}{12}+\frac{\sqrt{226}}{24} \approx 0.886$ and the matrix corresponds to an unstable method.

Our discussion helps to exclude non-skew symmetric matrices $B$ from further discussion yet, even if $B$ is skew symmetric, we are not done yet! Recall that the purpose of the exercise is to discretise $v(x) \partial / \partial x$, rather then merely $\partial / \partial x$. Thus, we assume that $v$ is a given smooth function, let $v_{m}=v(m \Delta x)$ and approximate $v_{m} u_{m}^{\prime}$ by multiplying the right-hand side of $(2.1)$ by $v_{m}, m=1, \ldots, M$. In other words, our interest is in the stability (for all 'nice' functions $v$ ) of the matrix $C$ such that $c_{m, k}=v_{m} b_{m, k}$, $m, k=1, \ldots, M$. Skew symmetry of $B$ is still necessary, of course, because of the trivial case $v \equiv 1$, but we cannot take for granted that it is sufficient for all functions $v$ of interest.

Theorem 1 Let the matrix $B$ be skew symmetric and banded, that is $b_{m, k}=0$ for $|m-k| \geq r+1$ for some $r \geq 1$, and let its coefficients be independent of $M$. Then the matrix $C$ is stable.

Proof Because of bandedness and skew symmetry of $B$, we have

$$
\tilde{c}_{m, k}= \begin{cases}0, & m=k \text { or }|m-k| \geq r+1, \\ \frac{1}{2}\left(v_{m}-v_{k}\right) b_{m, k}, & 1 \leq|m-k| \leq r .\end{cases}
$$

where $\tilde{C}=\frac{1}{2}\left(C+C^{\top}\right)$. We recall the Gerschgorin Theorem: The eigenvalues of the $M \times M$ matrix $A$ reside in the union of the discs

$$
\mathbb{S}_{m}[A]=\left\{z \in \mathbb{C}:\left|z-a_{m, m}\right| \leq \sum_{k \neq m}\left|a_{m, k}\right|\right\}, \quad m=1, \ldots, M .
$$

Therefore, $\tilde{C}$ being symmetric

$$
\mu[C] \leq \max \left\{x \in \mathbb{R} \cap \mathbb{S}_{m}[\tilde{C}]: m=1, \ldots, M\right\}=\max _{m=1, \ldots, M} \sum_{k \neq m}\left|\tilde{c}_{m, k}\right| .
$$

However, since $|v(x)-v(y)| \leq\left\|v^{\prime}\right\|_{\infty}|x-y|$,

$$
\begin{aligned}
\sum_{k \neq m}\left|\tilde{c}_{m, k}\right| & \leq \frac{1}{2} \sum_{|m-k| \leq r}\left|v_{m}-v_{k}\right|\left|b_{m, k}\right| \leq \frac{1}{2}\left\|v^{\prime}\right\|_{\infty}(\Delta x) \sum_{|m-k| \leq r}|m-k| \cdot\left|b_{m, k}\right| \\
& \leq r \Delta x \max _{m, k}\left|b_{m, k}\right| .
\end{aligned}
$$

We deduce that there exists $\beta \geq 0$ such that $\mu[C] \leq \beta \Delta x$, therefore $\left\|\mathrm{e}^{n(\Delta t) /(\Delta x) C}\right\|$ is uniformly bounded for any $n \Delta t$ in a compact interval for $\Delta x \rightarrow 0$ and the matrix corresponds to a stable method. 
Remark We have assumed in the proof of the theorem that the Courant number is $\Delta t / \Delta x$, this being the natural choice once we approximate $v(x) \partial / \partial x$. In the context of solving the convection-diffusion equation (1.1) the right choice of the Courant number is governed by the imperative to discretise $\partial^{2} / \partial x^{2}$ and is $\Delta t /(\Delta x)^{2}$. This, however, makes absolutely no difference to the proof.

We have proved that, subject to mild conditions, we need skew-symmetry of the differentiation matrix and that skew symmetry is sufficient for stability. These extra conditions are important. In principle, we might be able to forego skew symmetry of $B$, provided that the $\kappa_{M}$ s tend to zero (effectively, that $B$ approaches a skewsymmetric matrix as $M \rightarrow \infty$ in an appropriate norm). Likewise, Theorem 1 need not be true once $B$ is not banded. However, all said and done, it is fair to require skew symmetry of the differentiation matrix while constructing effective splitting-type methods for equations like (1.1). This turns out to be a surprisingly difficult problem.

\section{An order barrier for skew-symmetric differentiation matrices}

Imposing skew symmetry on (2.1) results in

$$
u_{m}^{\prime} \approx \frac{1}{\Delta x}\left(a_{m} u_{0}-\sum_{k=1}^{m-1} b_{k, m} u_{k}+\sum_{k=m+1}^{M} b_{m, k} u_{k}+c_{m} u_{M+1}\right), \quad m=1,2, \ldots, M,
$$

where we set $a_{m}=b_{m, 0}, c_{m}=b_{m, M+1}$ to emphasise the distinct role of boundary conditions. Order $p$ conditions are clearly

$$
a_{m} 0^{s}-\sum_{k=1}^{m-1} k^{s} b_{k, m}+\sum_{k=m+1}^{M} k^{s} b_{m, k}+(M+1)^{s} c_{m}=s m^{s-1}, \quad s=0, \ldots, p,
$$

for $m=1, \ldots, M$. We utilise the boundary coefficients $\left\{a_{m}\right\}$ and $\left\{b_{m}\right\}$ to satisfy $(3.2)$ for $s=0,1$. Thus, $s=0$ yields

$$
a_{m}=\sum_{k=1}^{m-1} b_{k, m}-\sum_{k=m+1}^{M} b_{m, k}-c_{m}, \quad m=1, \ldots, M,
$$

while $s=1$ results in

$$
c_{m}=\frac{1}{M+1}+\frac{1}{M+1} \sum_{k=1}^{m-1} k b_{k, m}-\frac{1}{M+1} \sum_{k=m+1}^{M} k b_{m, k}, \quad m=1, \ldots, M .
$$

The remaining $\frac{1}{2}(M-1) M$ coefficients of the matrix $B$ can be in principle used to obey (3.2) for higher values of $s$ and, naively counting degrees of freedom, we might expect that attaining high order with a skew-symmetric matrix $B$ should be possible. We will demonstrate in this section that this hope has no justification, indeed that (3.2) cannot be satisfied for $p=3$. 
We commence from $s=2$. (3.2) results in

$$
-\sum_{k=1}^{m-1} k^{2} b_{k, m}+\sum_{k=m+1}^{M} k^{2} b_{m, k}+(M+1)^{2} c_{m}=2 m, \quad m=1, \ldots, M,
$$

and substituting the value of $c_{m}$ from (3.3) yields

$$
\sum_{k=1}^{m-1} k(M+1-k) b_{k, m}-\sum_{k=m+1}^{M} k(M+1-k) b_{m, k}=2 m-M-1
$$

for all $m=1, \ldots, M$. We solve (3.4) for $b_{m, M}, m=1, \ldots, M-1$, the outcome being

$$
b_{m, M}=\frac{1}{M} \sum_{k=1}^{m-1} k(M+1-k) b_{k, m}-\frac{1}{M} \sum_{k=m+1}^{M-1} k(M+1-k) b_{m, k}+\frac{M+1-2 m}{M} .
$$

These values are substituted back into (3.4) for $m=M$. We obtain on the left-hand side

$$
\begin{aligned}
& \sum_{k=1}^{M-1} k(M+1-k) b_{k, M} \\
= & \frac{1}{M} \sum_{k=1}^{M-1} k(M+1-k)\left[\sum_{\ell=1}^{k-1} \ell(M+1-\ell) b_{\ell, k}-\sum_{\ell=k+1}^{M-1} \ell(M+1-\ell) b_{k, \ell}\right] \\
& +\frac{1}{M} \sum_{k=1}^{M-1} k(M+1-k)(M+1-2 k) \\
= & \frac{1}{M}\left[\sum_{k=2}^{M-1} \sum_{\ell=1}^{k-1} \ell(M+1-\ell) k(M+1-k) b_{\ell, k}\right. \\
& \left.\quad-\sum_{\ell=2}^{M-1} \sum_{k=1}^{\ell-1} k(M+1-k) \ell(M+1-\ell) b_{k, \ell}\right]+M-1=M-1,
\end{aligned}
$$

because the sums in the square brackets match each other. We recover the right-hand side of (3.4) and all is well: $p=2$ is satisfied.

We proceed in an identical manner with $s=3$, and this is the moment things turn sour. The order condition (3.2) is now

$$
-\sum_{k=1}^{m-1} k^{3} b_{k, m}+\sum_{k=m+1}^{M} b_{m, k}+(M+1)^{3} c_{m}=3 m^{2}, \quad, m=1, \ldots, M,
$$

and substituting (3.3) results in

$$
\sum_{k=1}^{m-1} k\left[(M+1)^{2}-k^{2}\right] b_{k, m}-\sum_{k=m+1}^{M} k\left[(M+1)^{2}-k^{2}\right] b_{m, k}=3 m^{2}-(M+1)^{2}
$$


for $m=1, \ldots, M$. Next, we substitute $b_{m, M}, m=1, \ldots, M-1$, from (3.5). After elementary algebra we obtain

$$
\begin{aligned}
& -\sum_{k=1}^{m-1} k(M-k)(M+1-k) b_{k, m}+\sum_{k=m+1}^{M-1} k(M-k)(M+1-k) b_{m, k} \\
= & 3 m^{2}-2 m(2 M+1)+M(M+1), \quad m=1, \ldots, M .
\end{aligned}
$$

For every $m=1, \ldots, M-2$ we solve (3.6) for $b_{m, M-1}$,

$$
\begin{aligned}
b_{m, M-1}= & \frac{1}{2(M-1)} \sum_{k=1}^{m-1} k(M-k)(M+1-k) b_{k, m} \\
& -\frac{1}{2(M-1)} \sum_{k=m+1}^{M-2} k(M-k)(M+1-k) b_{m, k} \\
& +\frac{1}{2(M-1)}\left[3 m^{2}-2 m(2 M+1)+M(M+1)\right], \quad m=1, \ldots, M-2 .
\end{aligned}
$$

Finally, we substitute this on the left-hand side of (3.6) for $m=M-1$ :

$$
\begin{aligned}
& -\sum_{k=1}^{M-2} k(M-k)(M+1-k) b_{k, M-1} \\
= & -\frac{1}{2(M-1)} \sum_{k=1}^{M-2} \sum_{\ell=1}^{k-1} k(M-k)(M+1-k) \ell(M-\ell)(M+1-\ell) b_{\ell, k} \\
& +\frac{1}{2(M-1)} \sum_{k=1}^{M-2} \sum_{\ell=m+1}^{M-2} k(M-k)(M+1-k) \ell(M-\ell)(M+1-\ell) b_{k, \ell} \\
& -\frac{1}{2(M-1)} \sum_{k=1}^{M-2} k(M-k)(M+1-k)\left[3 k^{2}-2 k(2 M+1)+M(M+1)\right] \\
= & -\frac{1}{2(M-1)} \sum_{k=2}^{M-2} \sum_{\ell=1}^{k-1} k(M-k)(M+1-k) \ell(M-\ell)(M+1-\ell) b_{\ell, k} \\
& +\frac{1}{2(M-1)} \sum_{\ell=2}^{M-2} \sum_{k=1}^{\ell-1} k(M-k)(M+1-k) \ell(M-\ell)(M+1-\ell) b_{k, \ell} \\
& +\frac{1}{24} M(M+1)(M+2) .
\end{aligned}
$$

The two sums above are identical once we swap indices, hence they cancel: the lefthand side is thus $\frac{1}{24} M(M+1)(M+2)$. The right-hand side of (3.6) for $m=M-1$ is however $5-3 M$ : it is easy to verify that they fail to match for all $M \geq 1$.

We have proved that

Theorem 2 The highest order of the skew-symmetric differentiation matrix given by (3.1) is two. 


\section{A third-order skew-symmetric differentiation matrix in a non-equidistant setting}

The main purpose of 'negative' results like Theorem 2 is to spur an interest in alternative settings. The most obvious manner to escape the setting of that theorem is by allowing a non-equidistant grid. Note that in that case, strictly speaking, we venture outside the conditions of Theorem 1 . However, the proof of the theorem can be easily extended to non-equidistant grids and the statement that skew-symmetry, in tandem with bandedness, leads to stability of the matrix $C$ remains true.

Let us assume that the number of grid points is even, $M=2 N$, and consider a mesh

$$
0=x_{0}<x_{1}<x_{2}<\cdots<x_{2 N}<x_{2 N+1}=1 .
$$

The underlying problem is symmetric with respect to $x=\frac{1}{2}$, the centre of the interval, and we impose similar symmetry on the mesh,

$$
x_{2 N+1-m}=1-x_{m}, \quad m=0,1, \ldots, N .
$$

The non-equidistant generalisation of (3.1) is

$u^{\prime}\left(x_{m}\right) \approx \alpha_{m} u(0)-\sum_{k=1}^{m-1} \beta_{k, m} u\left(x_{m}\right)+\sum_{k=m+1}^{2 N-1} \beta_{m, k} u\left(x_{k}\right)+\gamma_{m} u(1), \quad m=1, \ldots, 2 N-1$,

where the coefficients $\alpha_{m}, \beta_{m, k}$ and $\gamma_{m}$ depend upon $N$. The differentiation matrix

$$
\mathcal{D}_{1}=\left[\begin{array}{ccccc}
0 & \beta_{1,2} & \beta_{1,3} & \cdots & \beta_{1,2 N-1} \\
-\beta_{1,2} & 0 & \beta_{2,3} & \cdots & \beta_{2,2 N-1} \\
\vdots & \ddots & \ddots & \ddots & \vdots \\
-\beta_{1,2 N-2} & \cdots & -\beta_{2 N-3,2 N-2} & 0 & \beta_{2 N-2,2 N-1} \\
-\beta_{1,2 N-1} & \cdots & -\beta_{2 N-3,2 N-1} & -\beta_{2 N-2,2 N-1} & 0
\end{array}\right]
$$

is skew symmetric by design. The order- $p$ conditions, generalising (3.2), are

$\alpha_{m} 0^{s}-\sum_{k=1}^{m-1} \beta_{k, m} x_{k}^{s}+\sum_{k=m+1}^{2 N-1} \beta_{m, k} x_{k}^{s}+\gamma_{m}=s x_{m}^{s-1}, \quad s=0, \ldots, p, \quad m=1, \ldots, 2 N-1$.

To complete our general setting, we wish the coefficients in (4.1) to reflect the symmetry of the problem. Thus, we stipulate that

$\gamma_{m}=-\alpha_{2 N-m}, \quad \beta_{m, k}=\beta_{2 N-k, 2 N-m}, \quad k=m+1, \ldots, 2 N-1, \quad m=1, \ldots, 2 N-1$. 
For example, for $N=4$ the compound matrix $\left[\boldsymbol{\alpha}\left|\mathcal{D}_{1}\right| \boldsymbol{\gamma}\right]$ reads

$$
\left[\begin{array}{c|cccccccc|c}
\alpha_{1} & 0 & \beta_{1,2} & \beta_{1,3} & \beta_{1,4} & \beta_{1,5} & \beta_{1,6} & \beta_{1,7} & \beta_{1,8} & -\alpha_{8} \\
\alpha_{2} & -\beta_{1,2} & 0 & \beta_{2,3} & \beta_{2,4} & \beta_{2,5} & \beta_{2,6} & \beta_{2,7} & \beta_{1,7} & -\alpha_{7} \\
\alpha_{3} & -\beta_{1,3} & -\beta_{2,3} & 0 & \beta_{3,4} & \beta_{3,5} & \beta_{3,6} & \beta_{2,6} & \beta_{1,6} & -\alpha_{6} \\
\alpha_{4} & -\beta_{1,4} & -\beta_{2,4} & -\beta_{3,4} & 0 & \beta_{4,5} & \beta_{3,5} & \beta_{2,5} & \beta_{1,5} & -\alpha_{5} \\
\alpha_{5} & -\beta_{1,5} & -\beta_{2,5} & -\beta_{3,5} & -\beta_{4,5} & 0 & \beta_{3,4} & \beta_{2,4} & \beta_{1,4} & -\alpha_{4} \\
\alpha_{6} & -\beta_{1,6} & -\beta_{2,6} & -\beta_{3,6} & -\beta_{3,5} & -\beta_{3,4} & 0 & \beta_{2,3} & \beta_{1,3} & -\alpha_{3} \\
\alpha_{7} & -\beta_{1,7} & -\beta_{2,7} & -\beta_{2,6} & -\beta_{2,5} & -\beta_{2,4} & -\beta_{2,3} & 0 & \beta_{1,2} & -\alpha_{2} \\
\alpha_{8} & -\beta_{1,8} & -\beta_{1,7} & -\beta_{1,6} & -\beta_{1,5} & -\beta_{1,4} & -\beta_{1,3} & -\beta_{1,2} & 0 & -\alpha_{1}
\end{array}\right] .
$$

In other words, in addition to the axis of skew-symmetry along the main diagonal, we have an axis of symmetry along the par-diagonal of $\mathcal{D}_{1}$.

In this section we do not intend to explore general schemes of this kind, just to provide a single example of a scheme of order $p=3$, thereby demonstrating that the barrier of Theorem 2 can be breached by allowing a non-equidistant grid. To this end we choose a parameter $\rho \in(0,1 /(2 N)]$ and set

$$
x_{m}=m \rho, \quad x_{2 N+1-m}=1-m \rho, \quad m=0, \ldots, N .
$$

In other words, the grid is equidistant except for a single interval, $x_{N+1}-x_{N}=$ $1-2 N \rho$. In addition, we consider a quindiagonal matrix $\mathcal{D}_{1}$, in other words require that $\beta_{m, k}=0$ for $k-m \geq 3$. Letting

$$
\delta_{m}=\beta_{m, m+1}, \quad m=1, \ldots, 2 N-1, \quad \eta_{m}=\beta_{m, m+2}, \quad m=1, \ldots, 2 N-2,
$$

we recall the symmetry conditions

$$
\delta_{2 N-m}=\delta_{m}, \quad m=1, \ldots, N, \quad \eta_{2 N-m-1}=\eta_{m}, \quad m=1, \ldots, N-1 .
$$

Revisiting the case $N=4$, we have

$$
\left[\begin{array}{c|cccccccc|c}
\alpha_{1} & 0 & \delta_{1} & \eta_{1} & 0 & 0 & 0 & 0 & 0 & -\alpha_{8} \\
\alpha_{2} & -\delta_{1} & 0 & \delta_{2} & \eta_{2} & 0 & 0 & 0 & 0 & -\alpha_{7} \\
\alpha_{3} & -\eta_{1} & -\delta_{2} & 0 & \delta_{3} & \eta_{3} & 0 & 0 & 0 & -\alpha_{6} \\
\alpha_{4} & 0 & -\eta_{2} & -\delta_{3} & 0 & \delta_{4} & \eta_{3} & 0 & 0 & -\alpha_{5} \\
\alpha_{5} & 0 & 0 & -\eta_{3} & -\delta_{4} & 0 & \delta_{3} & \eta_{2} & 0 & -\alpha_{4} \\
\alpha_{6} & 0 & 0 & 0 & -\eta_{3} & -\delta_{3} & 0 & \delta_{2} & \eta_{1} & -\alpha_{3} \\
\alpha_{7} & 0 & 0 & 0 & 0 & -\eta_{2} & -\delta_{2} & 0 & \delta_{1} & -\alpha_{2} \\
\alpha_{8} & 0 & 0 & 0 & 0 & 0 & -\eta_{1} & -\delta_{1} & 0 & -\alpha_{1}
\end{array}\right] .
$$

It is now possible to produce explicitly coefficients $\alpha_{m}, \delta_{m}, \eta_{m}$ and the parameter $\rho$ such that the method is of order 3 and verify this by their direct substitution into the order conditions (4.2) for $p=3$. We endeavour to avoid this 'rabbit out of a hat' approach and instead present a constructive means of deriving the order-3 coefficients step by step. Note that, by virtue of symmetry, it is sufficient to verify the order conditions in the first half of the range, $m=1, \ldots, N$. Letting $\delta_{0}, \eta_{-1}, \eta_{0}=0$, the order conditions (4.2) for $s=0$ are

$$
\alpha_{m}-\eta_{m-2}-\delta_{m-1}+\delta_{m}+\eta_{m}-\alpha_{2 N+1-m}=0, \quad m=1, \ldots, N .
$$


Therefore

$$
\alpha_{m}=\eta_{m-2}+\delta_{m-1}-\delta_{m}-\eta_{m}-\alpha_{2 N+1-m}, \quad m=1, \ldots, N-1,
$$

while for $m=N$, because $\eta_{N}=\eta_{N-1}$, we have

$$
\alpha_{N}=\eta_{N-2}+\delta_{N-1}-\delta_{N}-\eta_{N-1}-\alpha_{N+1} .
$$

The $s=1$ condition is

$$
\alpha_{2 N+1-m}=-1-\eta_{m-2} x_{m-2}-\delta_{m-1} x_{m-1}+\delta_{m} x_{m+1}+\eta_{m} x_{m+2}, \quad m=1, \ldots, N,
$$

and, substituting the grid points (4.4) and distinguishing between different cases, we have

$$
\begin{aligned}
& \alpha_{2 N+1-m}=-1-(m-2) \rho \eta_{m-2}-(m-1) \rho \delta_{m-1}+(m+1) \rho \delta_{m}+(m+2) \rho \eta_{m} \\
& m=1, \ldots, N-2 \\
& \alpha_{N+2}=-1-(N-3) \rho \eta_{N-3}-(N-2) \rho \delta_{N-2}+N \rho \delta_{N-1}+(1-N \rho) \eta_{N-1} \\
& \alpha_{N+1}=-1-(N-2) \rho \eta_{N-2}-(N-1) \rho \delta_{N-1}+(1-N \rho) \delta_{N} \\
& \\
& \quad+[1-(N-1) \rho] \eta_{N-1} .
\end{aligned}
$$

The $s=2$ condition being

$-\eta_{m-2} x_{m-2}^{2}-\delta_{m-1} x_{m-1}^{2}+\delta_{m} x_{m+1}^{2}+\eta_{m} x_{m+2}^{2}-\alpha_{2 N+1-m}=2 x_{m}, \quad m=1, \ldots, N$, substitution of $\alpha_{2 N+1-m}$ from (4.7) gives, after some beautification,

$$
\begin{aligned}
& x_{m-2}\left(1-x_{m-2}\right) \eta_{m-2}+x_{m-1}\left(1-x_{m-1}\right) \delta_{m-1}-x_{m+1}\left(1-x_{m+1}\right) \delta_{m} \\
& -x_{m+2}\left(1-x_{m+2}\right) \eta_{m}=2 x_{m}-1, \quad m=1, \ldots, N
\end{aligned}
$$

and (4.4) yields, after straightforward algebra,

$$
\begin{gathered}
(m+1) \rho[1-(m+1) \rho] \delta_{m}=1-2 m \rho+(m-2) \rho[1-(m-2) \rho] \eta_{m-2} \\
-(m+2) \rho[1-(m+2) \rho] \eta_{m}+(m-1) \rho[1-(m-1) \rho] \delta_{m-1}, \\
m=1, \ldots, N-2, \\
N \rho(1-N \rho) \delta_{N-1}=1-2(N-1) \rho+(N-3) \rho[1-(N-3) \rho] \eta_{N-3} \\
-N \rho(1-N \rho) \eta_{N-1}+(N-2) \rho[1-(N-2) \rho] \delta_{N-2}, \\
N \rho(1-N \rho) \delta_{N}=1-N \rho+(N-2) \rho[1-(N-2) \rho] \eta_{N-2} \\
-(N-1) \rho[1-(N-1) \rho] \eta_{N-1}+(N-1) \rho[1-(N-1) \rho] \delta_{N-1} .
\end{gathered}
$$

This recursion for the $\delta_{m} \mathrm{~s}$ can be resolved explicitly and the following can be easily verified by substitution,

$$
\begin{aligned}
\delta_{m} & =\frac{1}{2 \rho}-\frac{m-1}{m} \cdot \frac{1-(m-1) \rho}{1-m \rho} \eta_{m-1}-\frac{m+2}{m+1} \cdot \frac{1-(m+2) \rho}{1-(m+1) \rho} \eta_{m}, \\
\delta_{N-1} & =\frac{1}{2 \rho}-\frac{N-2}{N-1} \cdot \frac{1-(N-2) \rho}{1-(N-1) \rho} \eta_{N-2}-\eta_{N-1}, \\
\delta_{N} & =\frac{1}{2 \rho} \frac{N+1}{N} \cdot \frac{1-(N+1) \rho}{1-N \rho}-2 \frac{N-1}{N} \cdot \frac{1-(N-1) \rho}{1-N \rho} \eta_{N-1} .
\end{aligned}
$$


Finally, we let $s=3$ in (4.2) and use (4.7) to eliminate the $\alpha_{2 N+1-m} \mathrm{~s}$, the outcome being

$$
\begin{aligned}
& x_{m-2}\left(1-x_{m-2}^{2}\right) \eta_{m-2}+x_{m-1}\left(1-x_{m-1}^{2}\right) \delta_{m-1}-x_{m+1}\left(1-x_{m+1}^{2}\right) \delta_{m} \\
& -x_{m+2}\left(1-x_{m+2}^{2}\right) \eta_{m}=3 x_{m}^{2}-1, \quad m=1, \ldots, N .
\end{aligned}
$$

Substituting the $\delta_{m}$ s from (4.9) results, after an elementary algebra, in the recurrences

$$
\begin{aligned}
& -(m-2)[1-(m-2) \rho] \eta_{m-2}+2 \frac{m^{2}-1}{m} \cdot \frac{[1-(m-1) \rho][1-(m+1) \rho]}{1-m \rho} \eta_{m} \\
& \quad-(m+2)[1-(m+2) \rho] \eta_{m}+1=0, \quad m=1, \ldots, N-2, \\
& -(N-3)[1-(N-3) \rho] \eta_{N-3}+2 \frac{(N-2) N}{N-1} \cdot \frac{[1-(N-2) \rho](1-N \rho)}{1-(N-1) \rho} \eta_{N-2} \\
& \quad-\frac{N}{\rho}(1-N \rho)(1-2 N \rho) \eta_{N-1}+1=0, \\
& -(N-2)[1-(N-2) \rho] \eta_{N-2}-\frac{N-1}{\rho}[1-(N-1) \rho](1-2 N \rho) \eta_{N-1} \\
& \quad-\frac{1}{\rho^{2}}\left[(N+1)-(N+1)(3 N+2) \rho+\left(2 N^{3}+5 N^{2}+4 N-1\right) \rho^{2}\right]=0 .
\end{aligned}
$$

The explicit solution, which can be easily verified by direct substitution, is surprisingly simple,

$$
\eta_{m}=\frac{m+1}{12} \cdot \frac{2-(m+1) \rho}{(1-m \rho)[1-(m+2) \rho]}, \quad m=1, \ldots, N-2,
$$

while we have two expressions for $\eta_{N-1}$, one originating in $m=N-1$ and the other in $m=N$,

$$
\begin{aligned}
\eta_{N-1} & =\frac{1}{12} \frac{(N+1) \rho(2-N \rho)}{[1-(N-1) \rho](1-N \rho)(1-2 N \rho)}, \\
& =\frac{1}{12} \frac{(N+1)\left[6-12(2 N+1) \rho+2\left(15 N^{2}+16 N-1\right) \rho^{2}-\left(12 N^{3}+19 N^{2}+N-2\right) \rho^{3}\right]}{(N-1) \rho[1-(N-1) \rho](1-N \rho)(1-2 N \rho)} .
\end{aligned}
$$

Equating these expressions results in a cubic equation for the parameter $\rho$,

$$
(2 N+1)\left(3 N^{2}+3 N-1\right) \rho^{3}-15 N(N+1) \rho^{2}+6(2 N+1) \rho-3=0 .
$$

This equation has two complex conjugate roots which are of no further interest, as well as a single real root whose asymptotic expansion can be easily verified by direct substitution,

$$
\rho=\frac{1}{2 N}-\frac{1}{4 N^{2}}-\frac{5}{24 N^{3}}+\frac{7}{16 N^{4}}+\cdots, \quad N \gg 1 .
$$

Note that it obeys the inequality $\rho \leq 1 /(2 N)$, as required, by a small margin, and we have

$$
\begin{aligned}
& x_{m+1}-x_{m}=\rho \approx \frac{1}{2 N}-\frac{1}{4 N^{2}}+\mathcal{O}\left(N^{-3}\right), \quad m \neq N, \\
& x_{N+1}-x_{N}=1-2 N \rho \approx \frac{1}{2 N}+\frac{5}{12 N^{2}}+\mathcal{O}\left(N^{-3}\right)
\end{aligned}
$$


- the departure from an equidistant grid is just $\mathcal{O}\left(N^{-2}\right)$ but, remarkably, it is sufficient to overcome the barrier of Theorem 2.

Substituting (4.10) and (4.11) into (4.9), we obtain

$$
\begin{aligned}
\delta_{m} & =\frac{1}{6 \rho} \cdot \frac{[1-(2 m+1) \rho][3-(2 m+1) \rho]}{(1-m \rho)[1-(m+2) \rho]}, \quad m=1, \ldots, N-2, \\
\delta_{N-1} & =\frac{1}{12 \rho} \cdot \frac{6-(26 N-10) \rho+\left(35 N^{2}-31\right) N \rho^{2}-\left(14 N^{2}-19 N+3\right) N \rho^{3}}{[1-(N-1) \rho](1-N \rho)(1-2 N \rho)}, \\
\delta_{N} & =\frac{1}{6 N \rho} \cdot \frac{3-(3 N+12) \rho+\left(15 N^{2}+7 N+2\right) \rho^{2}-\left(6 N^{2}+5 N+1\right) N \rho^{3}}{(1-N \rho)^{2}(1-2 N \rho)} .
\end{aligned}
$$

Next we go back to (4.7) to recover the $\alpha_{2 N+1-m} \mathrm{~s}$ for $m=1, \ldots, N$,

$$
\begin{aligned}
& a_{n+1-m}=\frac{\rho^{2}\left(1-\rho^{2}\right)}{[1-(m-2) \rho][1-(m-1) \rho](1-m \rho)[1-(m+1) \rho][1-(m+2) \rho]}, \\
& m=1, \ldots, N-2 \text {, } \\
& a_{N+2}=\frac{\rho}{12[1-(N-3) \rho][1-(N-2) \rho][1-(N-1) \rho](1-N \rho)}[2(N+1) \\
& -(N-2)(7 N+11) \rho+(N+1)\left(7 N^{2}-27 N+24\right) \rho^{2} \\
& \left.-(N-3)(N-2)(2 N+1) \rho^{3}\right] \text {, } \\
& a_{N+1}=\frac{1}{12 \rho N[1-(N-2) \rho][1-(N-1) \rho](1-N \rho)(1-2 N \rho)}[6(N+1) \\
& -6(N+1)(7 N-2) \rho+2(N+1)\left(57 N^{2}-40 N-1\right) \rho^{2} \\
& -N\left(150 N^{3}-39 N^{2}-175 N+2\right) \rho^{3}+\left(96 N^{5}-90 N^{4}-135 N^{3}\right. \\
& \left.\left.+27 N^{2}+20 N+8\right) \rho^{4}-2(N-2)(N-1) N(2 N+1)^{2}(3 N+1) \rho^{5}\right] .
\end{aligned}
$$

Finally, we substitute in (4.6) to derive $\alpha_{m}, m=1, \ldots, N$, in an explicit form and complete the description of the method,

$$
\begin{aligned}
& \alpha_{1}=-\frac{1}{6} \frac{3-\rho}{\rho}, \\
& \alpha_{2}=-\frac{1}{12} \frac{2-\rho}{1-2 \rho}, \\
& \alpha_{m}= 0, \quad m=3, \ldots, N-2, \\
& \alpha_{N-1}=-\frac{1}{12} \frac{(2-N \rho)[1-(2 N+1) \rho]}{[1-(N-1) \rho](-N \rho)}, \\
& \alpha_{N}=\frac{1}{12 \rho[1-(N-1) \rho](1-N \rho)^{2}(1-2 N \rho)}\left[6-(42 N+4) \rho+\left(114 N^{2}+19 N-5\right) \rho^{2}\right. \\
& \quad-\left(150 N^{3}+33 N^{2}+22 N+3\right) \rho^{3}[1-(N-1) \rho](1-N \rho)^{2}(1-2 N \rho) \\
& \quad+\left(96 N^{4}+26 N^{3}-33 N^{2}-15 N-4\right) \rho^{4} \\
&\left.\quad-2(N-1) N(2 N+1)^{3}(3 N+1) \rho^{5}\right] .
\end{aligned}
$$


$\alpha$
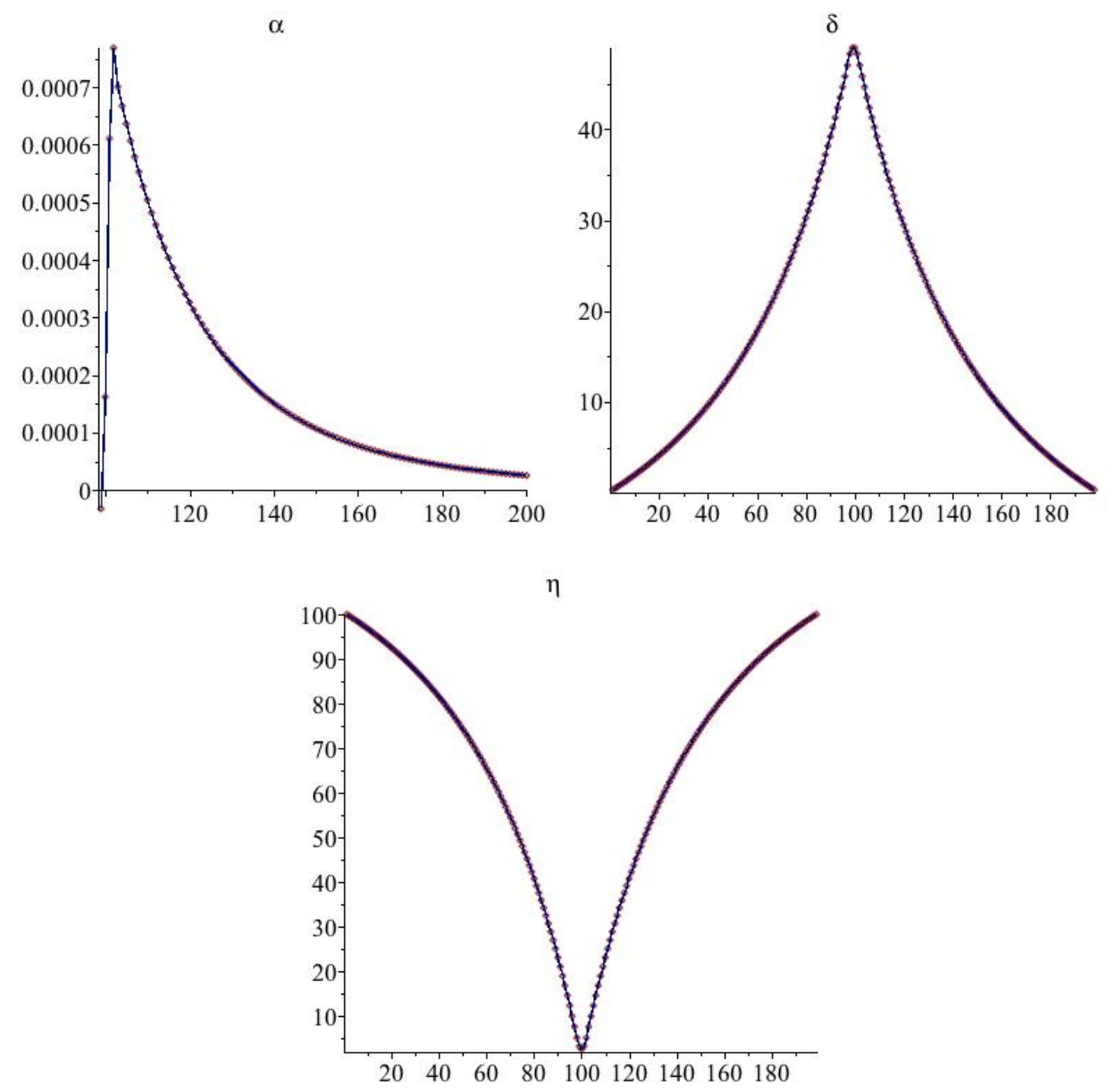

Figure 4.1: The coefficients $\alpha_{m}$ (for $\left.m=N-1, \ldots, 2 N\right), \delta_{m}, m=1, \ldots, 2 N$, and $\eta_{m}$, $m=1, \ldots, 2 N-1$, for $N=100$. (We did not plot the $\alpha_{m}$ s for $m \leq N-2$ because they are all zero, except for $\alpha_{1}$ and $\alpha_{2}$.)

In Fig. 4.1 we display the coefficients, as given by (4.10-14), for $N=100$ (in which case $\rho=0.00497479$ ). Note that their size varies fairly drastically: substituting the asymptotic expansion of $\rho$, we have

$$
\begin{aligned}
& \alpha_{1} \approx-\frac{N}{2}, \quad \alpha_{2} \approx-\frac{1}{6}, \quad \alpha_{m}=0, \quad m=3, \ldots, N-2, \quad \alpha_{N-1}, a_{N} \approx \frac{1}{2 N}, \\
& \alpha_{N+1} \approx-\frac{1}{6 N}, \quad \alpha_{N+2} \approx \frac{65}{2 N^{2}}, \quad \alpha_{m} \approx \frac{1}{N^{2}}, \quad m=N+3, \ldots, 2 N, \\
& \delta_{1}, \delta_{2 N-1} \approx \frac{N}{2}, \quad \delta_{m}, \delta_{2 N-m} \approx \frac{1}{2 N}-\frac{m}{3}, \quad m=2, \ldots, N-1,
\end{aligned}
$$




$$
\eta_{m}, \eta_{2 N-1-m} \approx \frac{m+1}{6}, \quad m=1, \ldots, N-2 .
$$

The variation in size of the coefficients is hardly a cause for concern. In the equidistant case the coefficients are scaled by $1 / \Delta x=\mathcal{O}(N)$ and we can expect the coefficients to be of similar order of magnitude in the present case. Indeed, a pleasing feature of the method is that the 'boundary coefficients' $\alpha_{m}$ are either zero or very small except for $m=1,2$, when the grid point where approximation takes place is near the relevant boundary. Note further that the size of the coefficients along each diagonal of $\mathcal{D}_{1}$ changes fairly drastically as $m$ varies: compare to the matrix (1.3) which is Toeplitz, i.e. constant along diagonals! The tiny, $\mathcal{O}\left(N^{-2}\right)$ departure from an equidistant setting, the very departure that allows us to breach the order barrier of Theorem 2, has a huge impact on the size of the coefficients!

What about higher orders? It is possible with significant extra effort to design a fourth-order method with a skew-symmetric matrix by adding to $\mathcal{D}_{1}$ an extra diagonal,

$\kappa_{m}=b_{m, m+3}$. In that case we are a single degree of freedom short of fulfilling fourthorder conditions with the grid (4.4). Instead we may consider the grid $x_{m}=m \rho$, $m=1, \ldots, K, x_{m}=m \sigma, m=K+1, \ldots, N$ for some $K \leq N-1$. Not all values of $K$ result in a 'proper' grid, for which $x_{m+1}-x_{m}>0$ for all $m=0, \ldots, 2 N$ and, in the absence of an overarching theory, we have just experimented with few values. Fig. 4.2 displays the coefficients for $K=34, N=100$ : in that case $\rho \approx 0.004974747$, $\sigma \approx 0.004971101$ (again, the deviation from an equidistant setting is very minor indeed: the exceptional intervals are $x_{K+1}-x_{K} \approx 0.005098723$ and $x_{N+1}-x_{N} \approx 0.005050588$ ). Moreover, $\alpha_{m}=0$ for $m=3, \ldots, K-3$. Note that all this is an outcome of a great deal of numerical experimentation. The numbers $(\rho, \sigma)$ are a solution of a bivariate high-degree polynomial system and typically there are many real solutions, although not all result in a proper grid. We have experimented with both $K$ and the freedom in choosing $\rho$ and $\sigma$ to minimise the deviation from an equidistant setting.

It is fair, however, to state that this sort of brute-force, hit-and-miss approach is not an adequate methodology in designing higher-order skew-symmetric differentiation matrices. Clearly, much better understanding of this fairly fundamental activity, approximating a derivative on a non-equidistant grid, is required.

\section{Conclusion}

The implementation of modern, high-order splitting methods for the convection-diffusion equation requires a suitably accurate approximation of the first derivative by a skew-symmetric matrix. In this paper we have proved that no third-order method of this kind is possible on an equidistant grid. Once non-equidistant grids are allowed, finite-difference methods of modestly higher orders can be constructed. However, the current state of the art leaves a great deal to be desired and considerably more effort is required to obtain high-order finite-difference-based differentiation matrices in a form amenable to efficient computation.

The temptation is to abandon finite differences altogether and seek a spectrallyconvergent basis with a skew-symmetric differentiation matrix. However, while for periodic boundary conditions such matrices abound - although, to be fair to finite differences, it is also trivial to construct skew-symmetric finite-difference differentiation 

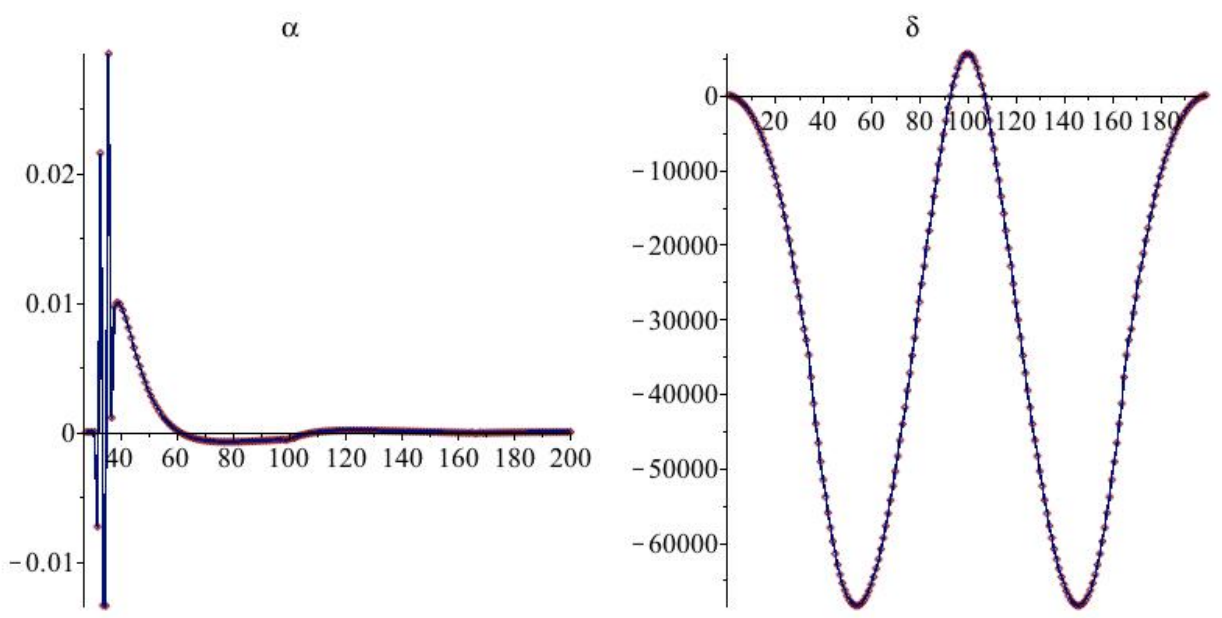

$\eta$
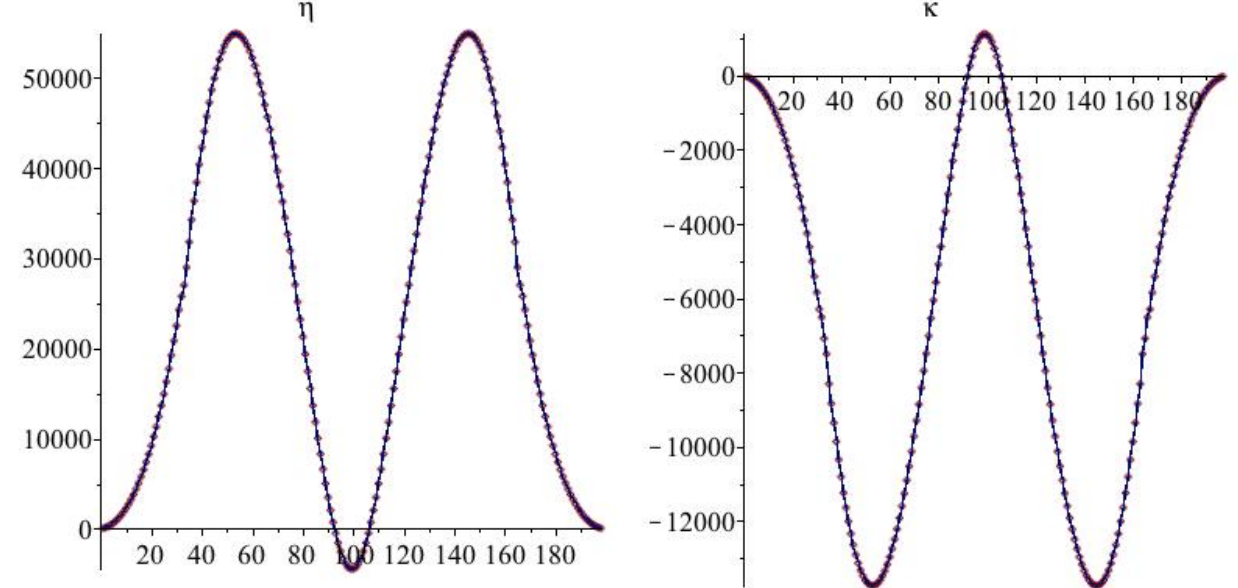

Figure 4.2: The fourth-order method: The coefficients $\alpha_{m}$ (for $m=K-2, \ldots, 2 N$ ), $\delta_{m}, \eta_{m}$ and $\kappa_{m}$ for $N=100$. (We did not plot the $\alpha_{m}$ s for $m \leq K-3$ because they are all zero, except for $\alpha_{1}, \alpha_{2}$ and $\alpha_{3}$.)

matrices in the periodic-boundary case - this is no longer true once the boundary conditions are Dirichlet. In that case ongoing research demonstrates that, while it is possible to choose a basis for $\mathrm{L}_{2}[-1,1]$ which yields a skew-symmetric differentiation matrix, it is not yet possible to compute fast and efficiently in that basis, thereby inheriting the wider benefits of a spectral method. This clearly is an issue deserving of much further research. 


\section{References}

Bader, P., Iserles, A., Kropielnicka, K. \& Singh, P. (2012), Effective approximation for the linear time-dependent Schrödinger equation, Technical report, DAMTP Tech. Rep. 2012/NA05, University of Cambridge.

Bejan, A. (2004), Convection Heat Transfer, 3rd edn, Wiley, New York.

Hairer, E., Lubich, C. \& Wanner, G. (2006), Geometric Numerical Integration, 2nd edn, Springer-Verlag, Berlin.

Hansen, E. \& Ostermann, A. (2009), 'High order splitting methods for analytic semigroups exist', BIT 49, 527-542.

Hesthaven, J., Gottlieb, S. \& Gottlieb, D. (2007), Spectral Methods for TimeDependent Problems, Cambridge University Press, Cambridge.

Hochbruck, M. \& Ostermann, A. (2010), 'Exponential integrators', Acta Numerica 19, 209-286.

Iserles, A. (1992), 'Rational approximants and numerical methods for initial-value problems', Physica D 60, 280-292.

Iserles, A. (2008), A First Course in the Numerical Analysis of Differential Equations, 2nd edn, Cambridge University Press, Cambridge.

McLachlan, R. I. \& Quispel, G. R. W. (2002), 'Splitting methods', Acta Numerica 11, 341-434.

Morton, K. W. (1996), Numerical Solution of Convection-Diffusion Problems, Chapman \& Hall, London.

Richtmyer, R. D. \& Morton, K. W. (1967), Difference Methods for Initial-Value Problems, 2nd edn, Wiley-Interscience, New York.

Sheng, Q. (1989), 'Solving linear partial differential equations by exponential splitting', IMA J. Numer. Anal. 9, 199-212.

Söderlind, G. (2006), 'The logarithmic norm. History and modern theory', BIT 46, 631-652. 